\title{
Simultaneous SAXS and SANS Analysis Detects Toroidal Supramolecular Polymers Composed of Noncovalent Supermacrocycles in Solution
}

\author{
Martin J. Hollamby, ${ }^{*[\mathrm{a}]}$ Keisuke Aratsu ${ }^{[\mathrm{b}]}$ Brian R. Pauw,${ }^{[\mathrm{c}]}$ Sarah E. Rogers, ${ }^{[\mathrm{d}]}$ Andrew J. Smith, ${ }^{[\mathrm{e}]}$ \\ Mitsuaki Yamauchi, ${ }^{[b]}$ Xu Lin ${ }^{[b]}$ Shiki Yagai ${ }^{*}{ }^{[b]}$
}

\begin{abstract}
Molecular self-assembly primarily occurs in solution. To better understand this process, techniques capable of probing the solvated state are consequently required. Small-angle scattering (SAS) has a proven ability to detect and characterise solutions, but is rarely applied to more complex assembly shapes. Here, we apply small-angle X-ray and neutron scattering to observe toroidal assemblies in solution. Combined analysis confirms that the toroids have a core-shell structure, with a $\pi$-conjugated core and an alkyl shell into which solvent penetrates. The dimensions determined by SAS agree well with those obtained by (dried-state) atomic force microscopy. Increasing the number of naphthalene units in the molecular building block yields greater rigidity, as evidenced by a larger toroid and a reduction in solvent penetration into the shell. The detailed structural analysis demonstrates the applicability of SAS to monitor complex solution-based self-assembly.
\end{abstract}

Self-assembly processes range from the micellisation of amphiphiles ${ }^{[1-3]}$ to supramolecular polymerization ${ }^{[4]}$ and onwards to the more elaborate folding of DNA origami. ${ }^{[5]}$ When apolar molecules associate, solvent-solvent and solvent-solute interactions play a key role in the assembly process. ${ }^{[6]}$ Upon solvent removal, the assembly structure is rarely unaffected, necessitating the probing of the sample structure in solution in order to form an accurate assembly model. Unfortunately, this is often difficult to do, and, while cryo-electron microscopy can reveal high-resolution structures in (frozen) solution, ${ }^{[1,7]}$ there are only a few reports of its use in non-aqueous solutions. ${ }^{[2]}$ Conversely, small-angle scattering (SAS) techniques using $X$ rays $(\mathrm{SAXS})^{[8]}$ or neutrons (SANS) ${ }^{[9]}$ can detect molecular assemblies with dimensions typically between $1-100 \mathrm{~nm}$ in

[a] Dr. M. J. Hollamby

School of Physical and Geographical Sciences

Keele University

Keele, Staffordshire, ST55BG, (UK)

E-mail: m.hollamby@keele.ac.uk

[b] K. Aratsu, M. Yamauchi, Dr. X. Lin, Prof. Dr. S. Yagai,

Graduate School of Engineering

Chiba University

1-33 Yayoi-cho, Inage-ku, Chiba 263-8522 (Japan)

E-mail: yagai@faculty.chiba-u.jp

[c] Dr. B. R. Pauw

BAM Federal Institute for Materials Research and Testing

Unter den Eichen 87, 12205 Berlin (Germany)

[d] Dr. S. E. Rogers

ISIS-STFC

Rutherford Appleton Laboratory

Chilton, Oxon OX11 OQX (UK)

[e] Dr. A. J. Smith

Diamond Light Source Ltd

Diamond House, Harwell Science and Innovation Campus, Didcot, Oxfordshire OX11 ODE, (UK)

Supporting information for this article (Experimental details including characterization of 2 plus SAXS and SANS data collection and analysis, UV/vis absorption and AFM data) is given via a link at the end of the document. solution. SAS results offer a bulk average over an appreciable volume, are dominated by the most commonly existing assembly structure, and therefore have a high statistical relevancy. SAXS and SANS are routinely used to probe various supramolecular assemblies, including molecular capsules and tubes, ${ }^{[10,11]}$ micelles, ${ }^{[2,3,12,13]}$ vesicles, ${ }^{[14,15]}$ organogels, ${ }^{[2,16]}$ and protein tertiary structures. ${ }^{[17]}$ Combining SAXS and SANS can be particularly insightful. The SAXS signal arises from regions contrasting in electron density, while SANS (in this case) highlights ${ }^{1} \mathrm{H}$-rich regions of an assembly dispersed in a ${ }^{2} \mathrm{D}$-rich solvent. ${ }^{[9]}$ Therefore, for molecules comprising both $\pi$ conjugated ( $\mathrm{e}^{-}$-rich, ${ }^{1} \mathrm{H}$-poor) and aliphatic $\left({ }^{1} \mathrm{H}-\right.$ rich, $\mathrm{e}^{-}$-poor $)$ parts such as asphaltenes ${ }^{[18]}$ or alkyl-fullerene derivatives, ${ }^{[2]}$ the SAXS and SANS signals will derive from the $\pi$-conjugated-rich and aliphatic-rich parts of an assembly, respectively. In these cases, combined SAXS and SANS allows the detection not only of a particular assembly morphology in solution but also of the internal structure. In this regard, the unique self-assembly of $\mathbf{1}$ in apolar media is an attractive target for SAS investigations (Figure 1a). Molecule 1, consisting of barbituric acid hydrogenbonding units, a naphthalene body and minidendron aliphatic tail, self-assembles to form toroidal supramolecular polymers. ${ }^{[19]}$ Although uniform toroids have been visualized by atomic force microscopy (AFM) observation on highly oriented pyrolytic graphite (HOPG) upon solvent removal, direct evidence for formation of toroids in solution is lacking. X-ray diffraction analysis of the bulk liquid crystalline phase of $\mathbf{1}$ demonstrated the formation of hexameric hydrogen-bonded supermacrocycles (rosettes), which further stack to form a 2D rectangular columnar structure. $^{[19]}$ Here, using combined SAXS and SANS, we demonstrate that the rosettes form and self-assemble into uniform toroidal supramolecular polymers in solution.

To support the SAS analysis of the assemblies formed by $\mathbf{1}$, it was necessary to identify other compounds that similarly self-assemble into toroids, but with different morphological parameters. Compound 2, in which two naphthalene moieties are directly connected by the 2 - and 6 - positions, fulfils these requirements. Supramolecular polymers of 1 and 2 were prepared by cooling their hot methylcyclohexane solutions from 100 to $20{ }^{\circ} \mathrm{C}$. Temperature-dependent UV-vis measurements upon cooling showed a red-shift occurring in the absorption bands of both compounds, indicating a slipped cofacial stacking of the aromatic chromophores (Figure S1). The melting temperature $\left(T_{\mathrm{m}}\right)$ at which half of the molecules become aggregated was increased from $52{ }^{\circ} \mathrm{C}$ for 1 to $76{ }^{\circ} \mathrm{C}$ for 2 at a concentration of $1 \times 10^{-4} \mathrm{M}$ (Figure S2), suggesting the significant increase of the thermal stability of the latter due to an enhanced $\pi-\pi$ stacking interaction. ${ }^{[20,21]}$

AFM observation of supramolecular polymers of $\mathbf{1}$ and $\mathbf{2}$, transferred from solution to HOPG by spin-coating, showed the formation of toroids (Figure 1c, e). The average radii $R$ (Figure 3) were measured to be $7.6 \pm 0.7 \mathrm{~nm}$ for 1 and $13 \pm 1.2 \mathrm{~nm}$ for 2 (Table 1). The thickness, $T$ and cross-sectional width after 
a)

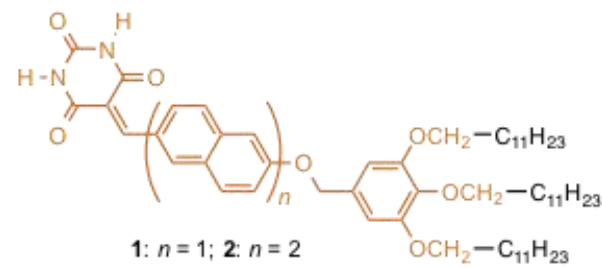

b)
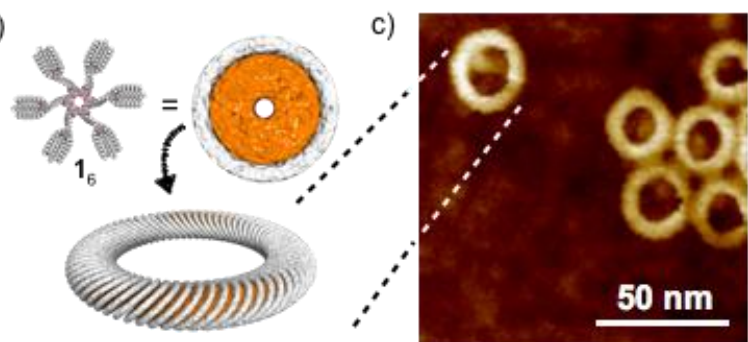

d)

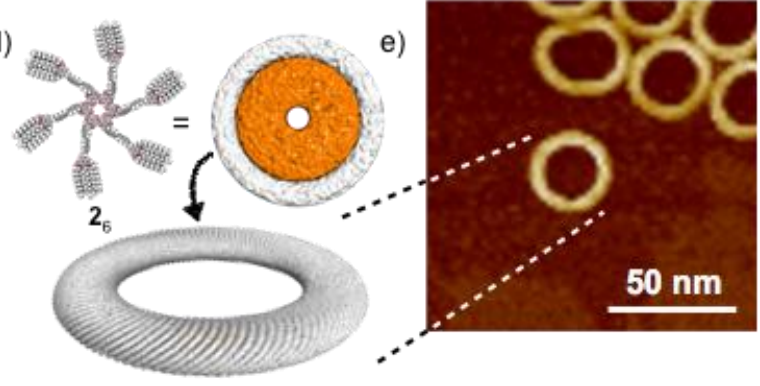

domains rich in alkyl chains. The simultaneous analysis of data with such differences in accentuation allows detailed information about the anticipated core-shell structure to be obtained.

The SANS and SAXS profiles obtained for $\mathbf{1}$ and $\mathbf{2}$ are shown in Figure 2. The positions of the features in the SAXS and SANS of the two compounds are similar, although the features in the data of $\mathbf{2}$ are shifted to lower $Q$ in comparison to $\mathbf{1}$, indicating that the overall assembly dimensions are larger. For
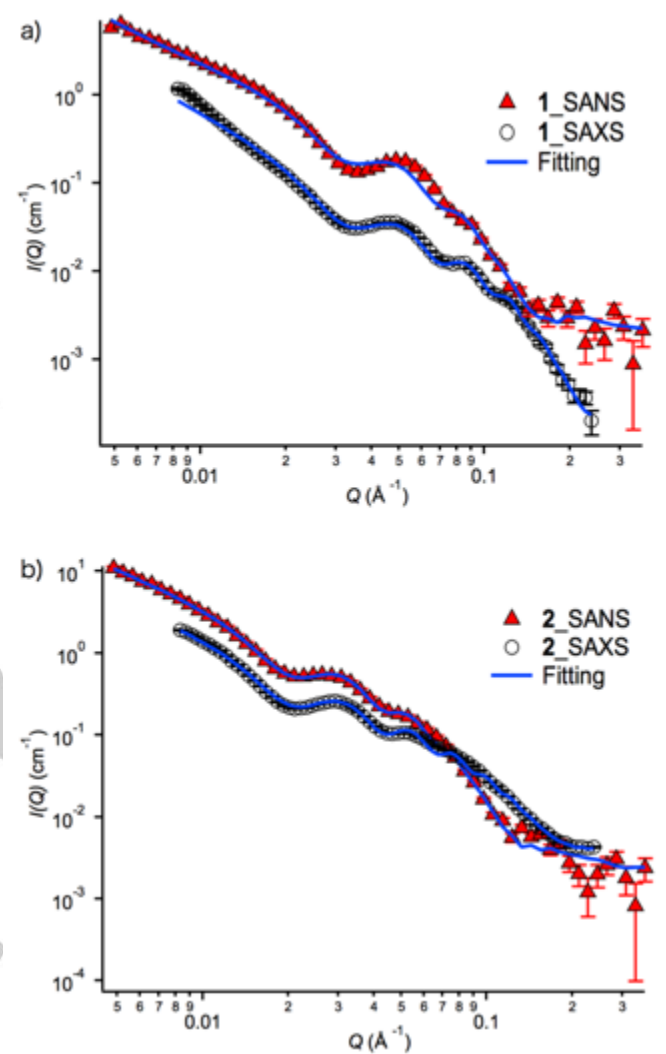

Figure 2. SANS (red triangles) and SAXS (open circles) data of a) 1 ( $c=1 \mathrm{mg}$ $\left.\mathrm{mL}^{-1}=1.08 \times 10^{-3} \mathrm{M}\right)$ and b) $2\left(c=1 \mathrm{mg} \mathrm{mL}^{-1}=0.95 \times 10^{-3} \mathrm{M}\right)$ in methylcyclohexane- $d_{14}$. Blue solid lines are fits to the data, using a toroid form factor and a flat background.

1 , and $T=2.7 \pm 0.4 \mathrm{~nm}$ and $W=5.6 \pm 2.0 \mathrm{~nm}$ for 2, respectively. Considering that these toroidal supramolecular polymers are formed by stacking of $C_{6}$ symmetrical rosettes, the ellipsoidal cross-section implies a tilted stacking of rosettes. These toroidal structures were observed over concentrations ranging from $1.0 \times 10^{-4}$ to $1.0 \times 10^{-3} \mathrm{M}$ for 1 , and from $5.0 \times 10^{-5}$ to $1.0 \times 10^{-3} \mathrm{M}$ for 2 (Figure S6). The lower concentrations are minimum limits below which we could not find toroids whereas at concentrations above $1.0 \times 10^{-3} \mathrm{M}$ it is difficult to recognize individual toroids due to heavy agglomeration.

To probe whether the toroids form in solution, supramolecular polymers of $\mathbf{1}$ and $\mathbf{2}$ prepared in methylcyclohexane- $d_{14}$ were analyzed by SANS and SAXS. The data exhibits several maxima and decays consistent with a toroidal form factor ${ }^{[22]}$ alongside an increase in scattering at low $Q$, which suggests a small degree of toroid-toroid aggregation likely arising from the relatively high sample concentration (see SI for details). The SAXS data is dominated by the higherelectron-density barbituric acid, naphthalene and benzyl parts of 1 and 2 in the lower electron density methylcyclohexane- $d_{14}$ matrix, while the SANS data originates predominantly from the both compounds, the decay in $/(Q)$ after the first peak in the middle of the $Q$-range $\left(Q_{\max }=0.05 \AA^{-1}\right.$ for $1, Q_{\max }=0.03 \AA^{-1}$ for 2) occurs more steeply for SANS than for SAXS. This relative difference is indicative of a core-shell structure..$^{[9]}$

To obtain more detailed information, the data has been modeled using a form factor for a monodisperse toroid with an ellipsoidal core-shell cross-section, ${ }^{[22]}$ with an attractive Ornstein-Zernike structure factor, $S(Q)$ accounting for the increased scattering at low $Q$ (see SI for details). ${ }^{[3]}$ A similar model without shell, has previously been used to model SANS data from toroidal charged block copolymer micelles, ${ }^{[23]}$ with others using hollow cylinders to approximate the toroidal structure. ${ }^{[10]}$ The dimensions observed by AFM were used as starting estimates for model fitting, and the scattering length densities of core, shell and solvent were estimated and fixed (see SI). SANS and SAXS data were fitted simultaneously using the multiple dataset fitting capability of the SASfit software ${ }^{[24]}$ to 
yield the dimensions $R$ (toroid radius), a (cross-section core radius), $\delta_{\text {shell }}$ (shell width), $b$ (cross-section aspect ratio) and the $S(Q)$ parameters. Fitting the data in this way affords far greater accuracy than is obtained by performing the fits independently.

The results of the fitting procedure have been added as blue solid lines to Figure 2, and selected analysis parameters are given in Table 1. The fitted core size is in line with those of molecular modeled structure of rosettes of 1 and 2 (Figure S3). For $\mathbf{2}, a$ is $5 \AA$ larger than for $\mathbf{1}$, which matches well with the length of a naphthalene unit $(4.9 \AA) \cdot{ }^{[25]}$ In line with the results from the temperature-dependent UV-vis and AFM measurements, SAS analysis points to an ellipsoidal crosssection, indicating that the rosettes pack in a tilted fashion into the supramolecular polymers. In solution, the tilt angle $\theta=$ $\arcsin (b)$ of the rosettes with respect to the toroid axis is $44^{\circ}$ for 1 and $38^{\circ}$ for 2 (see Figure 3 ). The difference in $b$ between SAS and AFM measurements for 2 can likely be ascribed to deformation of the toroidal nanostructures during the transition from a well-solvated state in solution to a dried and adsorbed state on the graphite substrate: the larger $\pi$-conjugated part in the rosette of $\mathbf{2}$ may bind more strongly to the graphite substrate, leading to greater disruption of the structure upon adsorption.
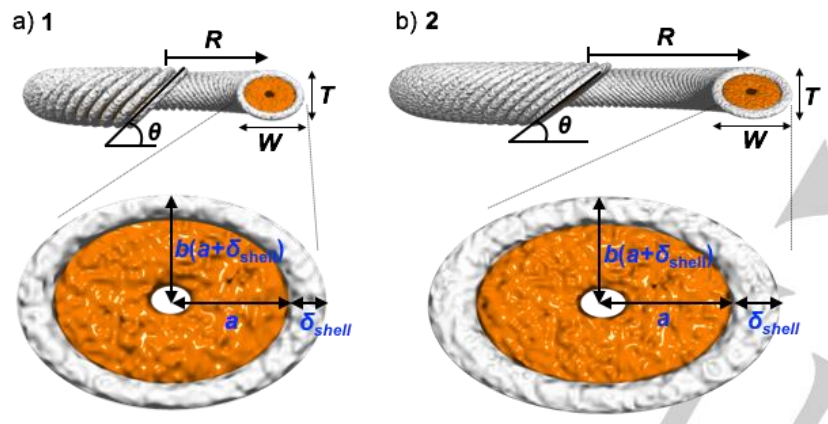

Figure 3. Schematic representations of the core-shell toroid of a) 1 and b) 2 with parameters obtained by AFM $(R, T$ and $W$ ) and model fitting of SAS data $\left.R, a, \delta_{\text {shell }}, b\right)$.

Table 1. Globally fitted parameters from modelling of SANS and SAXS data from $c=1 \mathrm{mg} \mathrm{mL}^{-1}$ solutions of $\mathbf{1}$ and $\mathbf{2}$ in methylcyclohexane-d $\mathrm{d}_{14}$ to a form factor of a toroid with a core-shell cross section, as shown schematically in Figure 1. The dimensions $R, a$ and $\delta_{\text {shell }}$ are $\pm 1 \AA$, while $b$ is \pm 0.01 .

\begin{tabular}{|c|c|c|c|c|}
\hline Parameters & SAS & AFM & SAS & AFM \\
\hline$R / \AA$ & 85 & 76 & 136 & 130 \\
\hline$a+\delta_{\text {shell }} / \AA$ & $\begin{array}{c}19(\mathrm{a}) \\
7\left(\delta_{\text {shell }}\right)\end{array}$ & & $\begin{array}{c}24(a) \\
10\left(\delta_{\text {shell }}\right)\end{array}$ & $28^{[\mathrm{a}]}$ \\
\hline$b$ & 0.70 & $0.71^{[\mathrm{b}]}$ & 0.62 & $0.47^{[b]}$ \\
\hline
\end{tabular}

[a] Equal to $W / 2$, where $W$ is the cross-sectional width. [b] Equal to $T / W$, where $T$ is the thickness.

The toroid radii, $R$ obtained by combined SANS and SAXS agree broadly with the AFM measurements (Figure 3). The toroids are larger for $\mathbf{2}$, which can be correlated with the enhanced $\pi-\pi$ interactions between the core, as demonstrated by temperature-dependent UV-vis analysis (Figure S2). The enhanced $\pi-\pi$ interactions increases the strength of interaction between the stacked rosettes, thus reducing the spontaneous curvature $\left(k=1 / R ; k_{1}=0.012 \AA^{-1}, k_{2}=0.0073 \AA^{-1}\right)$. More evidence for tighter packing in the assemblies formed by 2 is obtained from the fitted shell thickness, $\delta_{\text {shell. }}$ This is representative of the distance over which the alkyl chains remain unpenetrated by solvent, which is larger for 2 than for $\mathbf{1}$ : A larger $\delta_{\text {shell }}$ indicates a lower level of solvent penetration, in line with denser molecular packing within the rosette formed by 2 .

In conclusion, combined SAXS and SANS analysis confirms that 1 and 2 self-assemble into well-defined core-shell toroids in solution. The dimensions determined by SAS measurements and by AFM agree, although the assembly tends to deform and flatten when adsorbed and dried on graphite. This work adds to the growing evidence of the applicability of SAS measurements to elucidate the formation of complex shapes in solution via supramolecular self-assembly. Moreover, it highlights the power of combining SANS and SAXS analysis to obtain insight into the internal structure of complex supramolecular assemblies.

\section{Acknowledgements}

The authors acknowledge Diamond Light Source and the STFC for beam time on 122 and SANS2D, and consumables funding. This work was supported by KAKENHI (no. 26102010); a Grantin-Aid for Scientific Research on Innovative Areas " $\pi$-Figuration" (no. 26102001) of The Ministry of Education, Culture, Sports, Science, and Technology, Japan.

Keywords: nanorings $\cdot$ toroids $\cdot$ self-assembly $\bullet$ small-angle scattering • supramolecular chemistry

[1] S. Burghardt, A. Hirsch, B. Schade, K. Ludwig, C. Böttcher, Angew. Chem. Int. Ed. 2005, 44, 2976-2979.

[2] M. J. Hollamby, M. Karny, P. H. H. Bomans, N. A. J. M. Sommerdjik, A. Saeki, S. Seki, H. Minamikawa, I. Grillo, B. R. Pauw, P. Brown, et al., Nat. Chem. 2014, 6, 690-696.

[3] M. Kotlarchyk, S.-H. Chen, J. S. Huang, M. W. Kim, Phys. Rev. A 1984 29, 2054-2069.

[4] T. F. A. De Greef, M. M. J. Smulders, M. Wolffs, A. P. H. J. Schenning,

R. P. Sijbesma, E. W. Meijer, Chem. Rev. 2009, 109, 5687-5754.

[5] P. W. K. Rothemund, Nature 2006, 440, 297-302.

[6] L. Yang, C. Adam, G. S. Nichol, S. L. Cockroft, Nat Chem 2013, 5, 1006-1010.

[7] J. L. S. Milne, M. J. Borgnia, A. Bartesaghi, E. E. H. Tran, L. A. Earl, D. M. Schauder, J. Lengyel, J. Pierson, A. Patwardhan, S. Subramaniam, FEBS J 2013, 280, 28-45.

[8] B. R. Pauw, J. Phys.: Condens. Matter 2013, 25, 383201.

[9] M. J. Hollamby, Phys. Chem. Chem. Phys. 2013, 15, 10566-10579.

[10] H. Kumari, S. R. Kline, W. G. Wycoff, R. L. Paul, A. V. Mossine, C. A

Deakyne, J. L. Atwood, Angew. Chem. Int. Ed. 2012, 51, 5086-5091.

[11] H. Kumari, S. R. Kline, W. G. Wycoff, J. L. Atwood, Small 2012, 8 , 3321-3325.

[12] M. J. Hollamby, K. J. Trickett, A. Mohamed, S. Cummings, R. F. Tabor, O. Myakonkaya, S. Gold, S. Rogers, R. K. Heenan, J. Eastoe, Angew. Chem. Int. Ed. 2009, 48, 4993-4995.

[13] M. J. Hollamby, R. Tabor, K. J. Mutch, K. Trickett, J. Eastoe, R. K. Heenan, I. Grillo, Langmuir 2008, 24, 12235-12240.

[14] J. Gummel, M. Sztucki, T. Narayanan, M. Gradzielski, Soft Matter 2011 7, 5731-5738.

[15] M. Bergström, J. S. Pedersen, P. Schurtenberger, S. U. Egelhaaf, J. Phys. Chem. B 1999, 103, 9888-9897. 
[16] X. Huang, P. Terech, S. R. Raghavan, R. G. Weiss, J. Am. Chem. Soc. 2005, 127, 4336-4344.

[17] M. V. Petoukhov, D. I. Svergun, Eur Biophys J 2006, 35, 567-576.

[18] J. Eyssautier, P. Levitz, D. Espinat, J. Jestin, J. Gummel, I. Grillo, L. Barré, J. Phys. Chem. B 2011, 115, 6827-6837.

[19] S. Yagai, Y. Goto, X. Lin, T. Karatsu, A. Kitamura, D. Kuzuhara, H. Yamada, Y. Kikkawa, A. Saeki, S. Seki, Angew. Chem. Int. Ed. 2012, 51 6643-6647.

[20] S. Yagai, S. Kubota, H. Saito, K. Unoike, T. Karatsu, A. Kitamura, A. Ajayaghosh, M. Kanesato, Y. Kikkawa, J. Am. Chem. Soc. 2009, 131, 54085410.
[21] S. Yagai, Y. Goto, T. Karatsu, A. Kitamura, Y. Kikkawa, Chem. Eur. J.

2011, 17, 13657-13660.

[22] T. Kawaguchi, J. Appl. Cryst. 2001, 34, 580-584.

[23] S. Förster, N. Hermsdorf, W. Leube, H. Schnablegger, M. Regenbrecht S. Akari, P. Lindner, C. Böttcher, J. Phys. Chem. B 1999, 103, 6657-6668. [24] I. Breßler, J. Kohlbrecher, A. F. Thünemann, J. Appl. Cryst. 2015, 48 1587-1598.

[25] S. C. Abrahams, J. M. Robertson, J. G. White, Acta Cryst. 1949, 2 233-238. 
Entry for the Table of Contents (Please choose one layout)

Layout 1:

\section{COMMUNICATION}

Molecules comprising a barbituric acid hydrogen-bonding unit, naphthalene body and aliphatic tail self-assemble in solution into toroids. Using small-angle X-ray and neutron scattering, with complementary spectroscopic and microscopic data, a core-shell structure is revealed. This demonstrates the applicability of small-angle scattering analysis to complex solution-based selfassembled nanostructures.

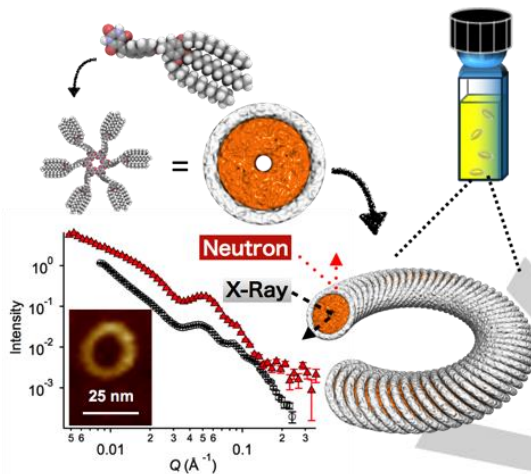

Martin J. Hollamby*, Keisuke Aratsu, Brian R. Pauw, Sarah E. Rogers, Andrew J. Smith, Mitsuaki Yamauchi, Xu Lin, Shiki Yagai*

Page No. - Page No.

Combined SAXS and SANS Analysis Detects the Circularized Supramolecular Polymerization of Noncovalent Supermacrocycles in Solution

Layout 2:

\section{COMMUNICATION}
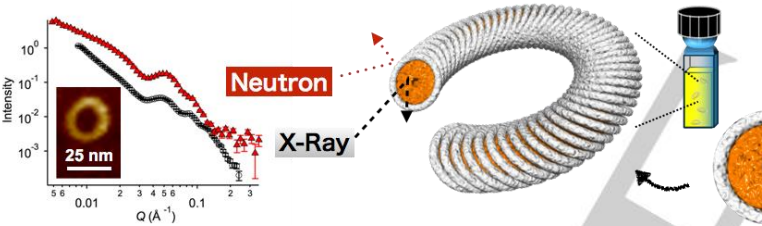

Molecules comprising a barbituric acid hydrogen-bonding unit, naphthalene body and aliphatic tail self-assemble in solution into toroids. Using small-angle X-ray and neutron scattering, with complementary spectroscopic and microscopic data, a core-shell structure is revealed. This demonstrates the applicability of small-angle scattering analysis to complex solution-based self-assembled nanostructures.
Martin J. Hollamby ${ }^{*}$, Keisuke Aratsu, Brian R. Pauw, Sarah E. Rogers, Andrew J. Smith, Mitsuaki Yamauchi, Xu Lin, Shiki Yagai*

Page No. - Page No.

Combined SAXS and SANS Analysis Detects the Circularized Supramolecular Polymerization of Noncovalent Supermacrocycles in Solution 